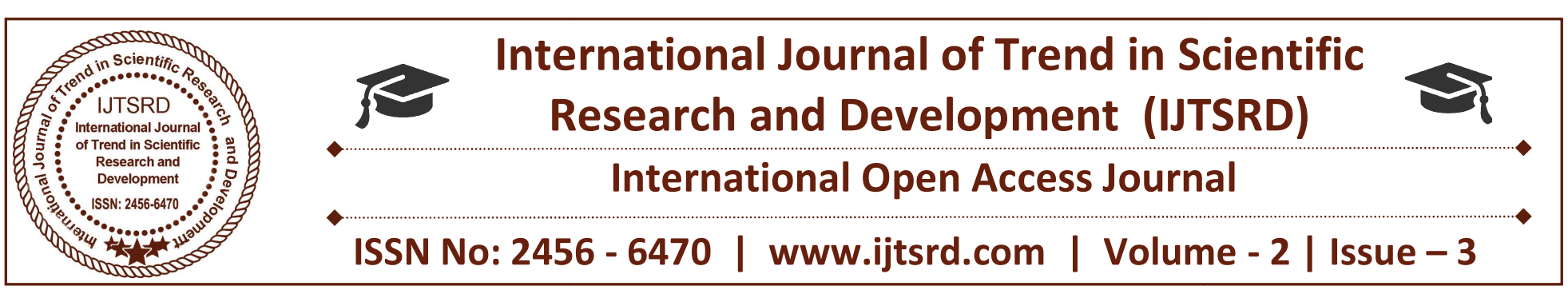

\title{
Fault Analysis Using Stft and Travelling Wave Method
}

\author{
Rajesh Saini \\ Department of Electrical \\ Engineering, Poornima College of \\ Engineering, Sitapura, Jaipur, \\ Rajasthan, India
}

\author{
Dr. Deepika Chauhan \\ Department of Electrical \\ Engineering, Poornima College of \\ Engineering, Sitapura, Jaipur, \\ Rajasthan, India
}

\author{
Mr. Brijraj Singh \\ Department of Electrical \\ Engineering, Poornima College of \\ Engineering, Sitapura, Jaipur, \\ Rajasthan, India
}

\section{ABSTRACT}

Current power supplies must give dependable, fantastic power. In this manner, it is vital that a transmission line blame be recognized precisely, dependably and rectified at the earliest opportunity. A strategy for arranging flaw compose in view of brief time Fourier change (STFT) is given in this paper. STFT is a methods for flag preparing in which a Fourier change (FT) is performed by setting a window work with a consistent size. This change is utilized to break down drifters in the power framework. This paper breaks down qualities of STFT by utilizing reproduction comes about because of MATLAB and characterizes line blames by blame kind. Precise and quick acknowledgment of transmission line blame area is of incredible criticalness to control framework security. Keeping in mind the end goal to settle the $\mathrm{T}$ compose transmission line blame area issue, the wave mode change and wavelet change are utilized to get the wavelet modulus maxima, and after that decide line overvoltage wave entry time, finally figure the blame area as per the blame area equation. This paper depicted the guideline of transmission line blame area in light of voyaging waves. The vulnerability of wave speed conveys blunder to blame area in light of voyaging waves. The main heartbeat wave of blame likewise gives numerous trademark data of the disappointment which was utilized to distinguish the sort of disappointments to advantageous the laborers making focused on surge repair.

Keywords: Short Time Fourier Transform, Transmission Line, EMTP, Fault Detection and Classification.

\section{INTRODUCTION}

Transmission lines are basic parts of electrical power framework. Line shortcomings may occur for various reasons including hardware breakdown, and lightning. An adjust blame is a 3-ø and an unbalance blame to a solitary line-to-ground blame (SLG), line-to-line cut off (DLL) or twofold line-to-ground blame (DLG). To shield control framework from these lacks, a figuring to distinguish diverse aggravations is required. Blame disclosure and request are essential to guaranteeing electrical power framework.

Fourier change (FT) is a strategy to trade a flag from the time space to the recurrence zone. In any case, the FT system has a drawback. When changing to the recurrence zone, the time information is lost. As an alternative, brief time Fourier change (STFT) can be used to evacuate information about the

Ampleness of the recurrence in the picked time territory by setting a moving window to an enduring size and sliding the moving window in the time space. Voyaging wave technique uses the blame partition and the spreading time to process the blame division. Impedance methodology and disillusionment examination are impacted by the structure parameters, the framework cut off insurance, the control arrange undertaking mode and so on, inciting the lower precision of region. In any case, the rule of voyaging wave strategy is essential, less impacted by the above factors. The discovered division, in any case, depends seriously on the scarcely getting multiplication speed of the beat wave, and the hang or the authentic length of the transmission lines, which may not be available as a general rule. 
International Journal of Trend in Scientific Research and Development (IJTSRD) ISSN: 2456-6470

\section{Need of Fault Analysis}

- Fast information about the sort and zone of blame can help created by help and blame room which constrain the fiscal effect of vitality interruption.

- Transmission and dispersion compose are the establishment of present influence framework and blame on this framework can cause outrageous budgetary disasters.

- An examination of framework disrupting impact gives a wealth of critical information concerning control framework ponders and the direct of security framework.

\section{Short Time Fourier Transform}

Brief time Fourier change (STFT) is a remarkable strategy for time-recurrence examination of a nonstationary flag. The explanation behind STFT is to get the time assortment in the recurrence substance of the flag. It alters the Fourier change (FT) to separate only a little fragment of the flag at a period a technique called windowing the flag. It can be spoken with a flag as a two-dimensional limit of time additionally, recurrence. The STFT addresses a tradeoff amongst time-and recurrence based flag perspectives. It gives a few information about both when and what events happen. Starting late, a couple of examinations have been performed on the ID and portrayal of inadequacies on transmission lines using diverse propelled flag getting ready (DSP) strategies. STFT, which is a standout amongst the most direct DSP mechanical assemblies, is every now and again used consequently. The fundamental objective of timerecurrence examination is to describe a limit that will depict the imperativeness thickness of a flag in the meantime with respect to time and recurrence.

With a ultimate objective to amend this issue, STFT is associated by moving a window of a steady size for a flag. Among the gadgets for separating signs, STFT is a champion among the most generally perceived and understood. The central rule of STFT is to cut up the flag into sensible covering time parcels (using the windowing strategy) and after that to play out a Fourier examination on each slice to find the frequencies contained in it. Recurrence information is connected with the time record in the midst of each cut of windowed data. In the region the STFT is described as in condition (1).

\section{Characteristics of Every Kind of Fault}

In the above figures characteristics of all kind of phases are shown which are based on simulation result like Single lint-ground (SLG) fault, line-line fault (DLL) and three phase fault.

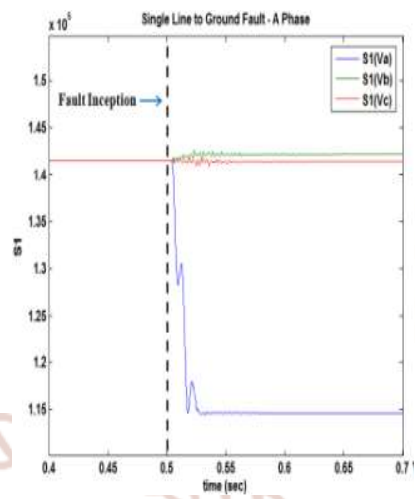

(a) SLG

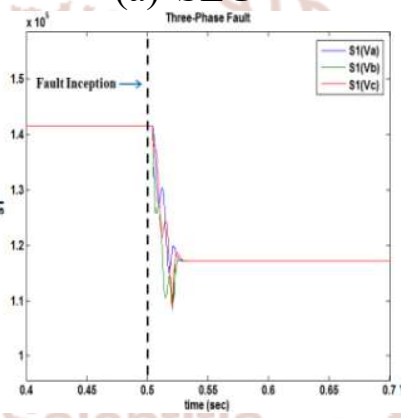

(b) Three phase fault (d) DLG

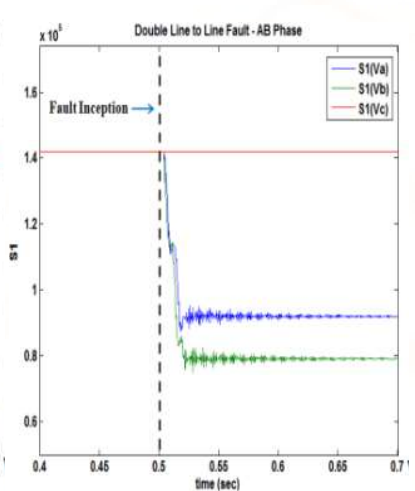

(b) DLF

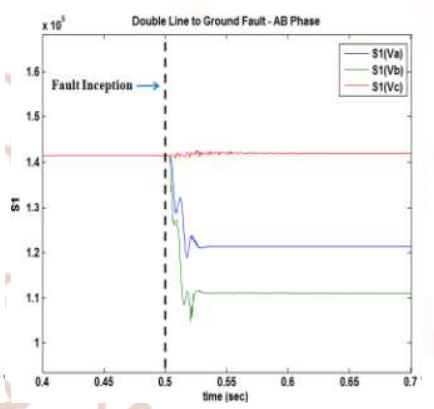

Figure 2: Variation in $\mathrm{S} 1$ according to the type of line fault

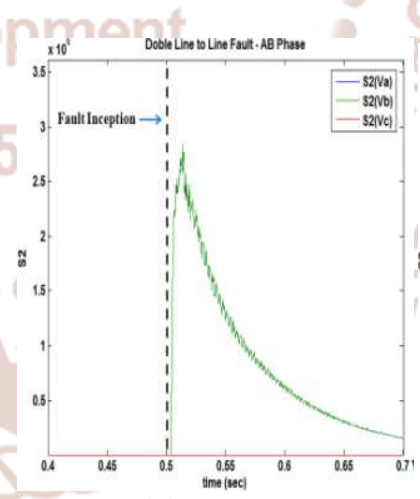

(a) DLF

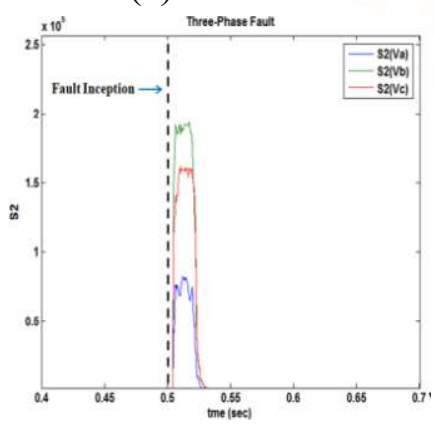

(c) Three phase fault

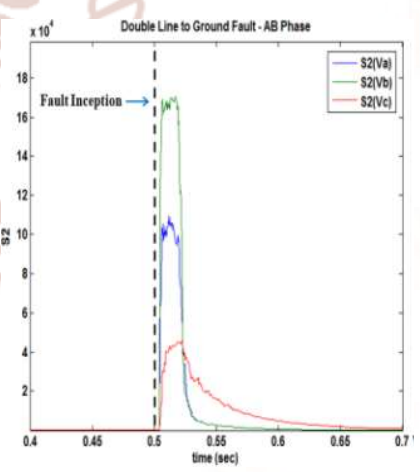

(b) DLG

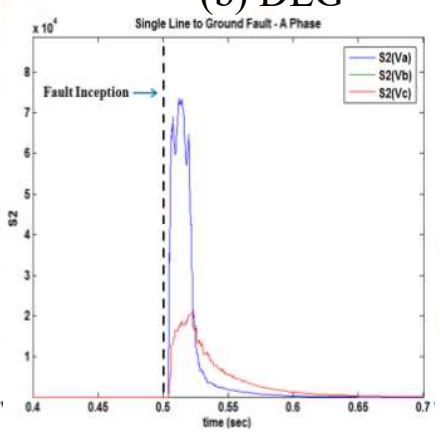

(d) SLG
Figure 3: Variation in S2 according to the type of line fault 
Figure 2 and Figure 3 are the consequence of a reproduction whenever SLG, DLL, DLG and the three-stage blame occur in the transmission line between Bus 1 and Bus 2 when the blame insurance is $50 \Omega$ blame beginning point is $90^{\prime}$ and the blame separation is $100 \mathrm{~km}$ from Bus 1 . In the wake of playing out the STFT for the voltage movement at the blame, line insufficiencies can be perceived through the change in waveform at the blame stage using S1 and S2 esteems. All things considered, by virtue of SLG, the voltage of the blame stage theoretically lessens. S1 demonstrates the modifications in the significance, which exhibits a similar example. After a line blame occurs at $0.5 \mathrm{~s}$, the stages don't change by and large. Of course, the S1 estimation of the blame voltage in the blame stage is by and large decreased after blame inception. By then the S1 esteem for the blame voltage settles at a lower than common esteem. S2 demonstrates an advancing consonant, which is commonly higher for the blame stage differentiated and sound stages. After a line blame happens, sound stages experience some assortment in the midst of one cycle. The S2 estimation of the blame stage is obviously extended differentiated and the strong stages and at last returns to the main esteem.

Right when a DLL blame happens, only the voltage of the blame stages reduce. The voltage of the strong stage remains reliable at the hidden quality. These characteristics are the same for the $\mathrm{S} 1$ esteem portrayed in Figure 2(b) and for the S2 esteem, which only additions in the blame stages. DLG resembles the DLL blame. With DLG, the S2 estimation of the strong stage and the blame stages increases to some degree, not under any condition like when a DLL blame happens. The assortment in the S1 and S2 esteems is showed up in Figure 2(c) and Figure 3(c), independently. For the three-stage blame, the voltage of all blame stages decreases rapidly and mightily. Assortments in the waveform in like manner appear differently in relation to blame assurance and stage. These characteristics are evident through the alteration in the S1 esteem delineated in Figure 2(d). The S2 esteem keeps an eye on increase for all blame stages after the line blame as showed up in Figure $3(d)$.

\section{Algorithm for Fault Classification using STFT}

In perspective of the qualities analyzed in Section 3, a figuring for gathering the kind of line blame is made using the STFT strategy around there. In Table 2, a conspicuous verification number is doled out to each blame kind. Figure 4 exhibits the estimation for requesting the blame kind using STFT

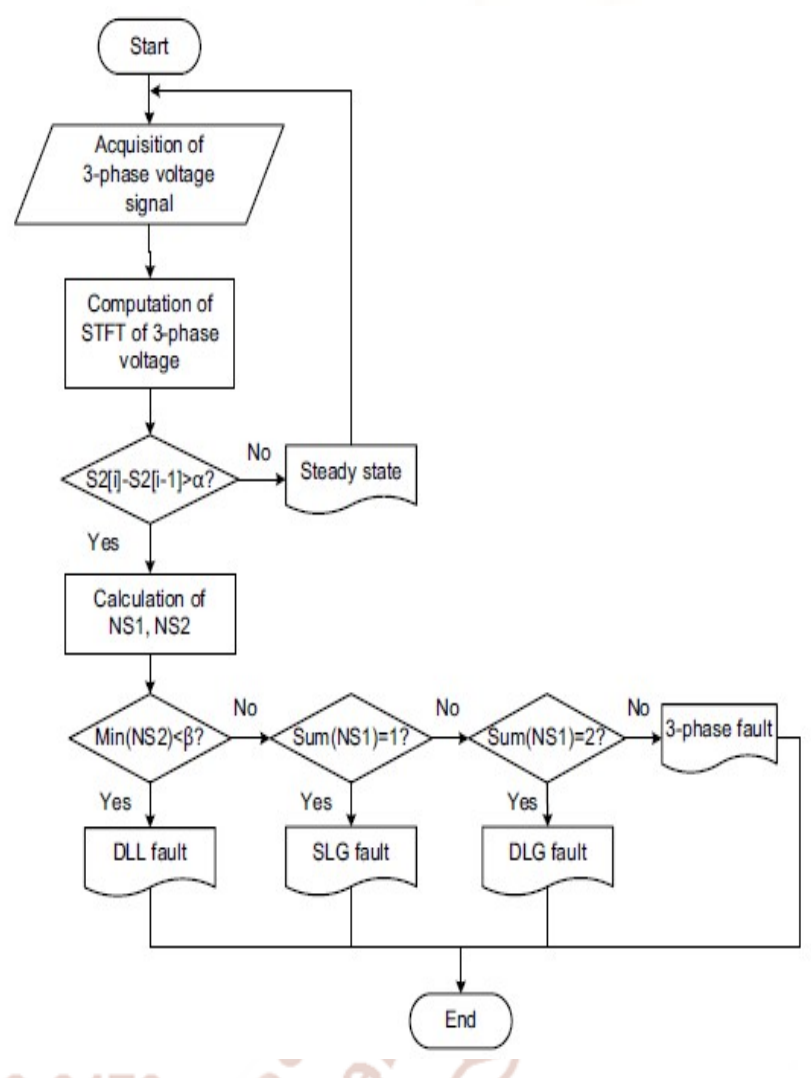

Figure 4: Algorithm for classifying the fault type

In Figure 4, the basic state of the prescribed computation is set to conventional. In any case, it achieves the three-eliminate voltages for playing the figuring and practices the STFT for the acquired voltages. The rate of assortment in the degree of the $\mathrm{S} 2$ esteem stood out from standard conditions is used to perceive the line blame. If S2 is run of the mill, the estimation comes back to the basic stage and a while later chooses the voltage iteratively. While distinguishing a line blame, it figures NS1 and NS2 at the same time in the midst of one cycle and subsequently perceives the sorts of line issues. This paper looks at how to mastermind inadequacies using the outcomes of the examination of the NS1 and NS2 characteristics in light of the blame sorts.

In the first place, qualities of the $\mathrm{S} 1$ and $\mathrm{S} 2$ esteems are dismembered in light of the line blame. S1 and S2 esteems remain relentless under customary conditions. Differentiations in S2 esteems are used to distinguish 
a line blame in light of the fact that $\mathrm{S} 2$ assortment is clearer than S1 assortment when line inadequacies happen. The most extraordinary estimation of S2 is around 1.6 and variable $\alpha$, which is the edge a motivating force for recognizing the line deficiencies, is set to 2 in light of the edge in the best differential esteem. The point of confinement esteem $\alpha$ can be changed by the condition of the power framework. Exactly when the total of NS1 esteems for each stage is 1, the blame sort is SLG, and if the aggregate of NS1 for each stage is 2, by then the blame kind is DLG. The breaking point esteem $\delta$ is set to 0.4 .

\begin{tabular}{|c|c|c|c|}
\hline TYPE & Fault Type & TYPE & Fault Type \\
\hline $\mathbf{1}$ & $\begin{array}{c}\text { Normal } \\
\text { State }\end{array}$ & 7 & DLG-BC phase \\
\hline $\mathbf{2}$ & $\begin{array}{c}\text { SLG-A } \\
\text { phase }\end{array}$ & 8 & DLL-AB phase \\
\hline $\mathbf{3}$ & $\begin{array}{c}\text { SLG-B } \\
\text { phase }\end{array}$ & 9 & DLL-AC phase \\
\hline $\mathbf{4}$ & $\begin{array}{c}\text { SLG-C } \\
\text { phase }\end{array}$ & 10 & DLL-BC phase \\
\hline $\mathbf{5}$ & $\begin{array}{c}\text { DLG-AB } \\
\text { phase }\end{array}$ & 11 & [ -fault Ina \\
\hline $\mathbf{6}$ & $\begin{array}{c}\text { DLG-AC } \\
\text { phase }\end{array}$ & & \\
\hline
\end{tabular}

\section{Limitation of Fourier Transform}

1. Unable to give time and recurrence area in the meantime.

2. Less well known for assessing time-variation, non-stationary signs.

3. Not a legitimate technique for speaking to discontinuities or sharp corner (i.e. requires a substantial number of Fourier parts to speak to discontinuities).

\section{Traveling Wave Method}

The essential systems are impedance methodology, the blame examination and voyaging wave technique. Impedance methodology registers the blame impedance by blame voltage and current after the blame happening, and a while later finds out the blame detachment by the estimation of the impedance; Failure examination figures the blame partition in light of the blame voltage and present as a component of the blame division; Traveling wave system uses the blame detachment and the spreading time to process the blame partition. Impedance methodology and dissatisfaction examination are impacted by the structure parameters, the framework cut off security, the power grid undertaking mode and so forth, inciting the lower precision of territory. Regardless, the standard of voyaging wave technique is essential, less affected by the above components. So the voyaging wave procedure is transforming into the key system for transmission line blame territory.

A. Voltage traveling wave signal phase transformation

After the transmission line blame happening, the overvoltage wave has spread to the two terminals of the line, and assembled by voltage acquisition contraption presented in the substation busbar in or close zenith. There are basic coupling and lacking transposition ponder between transmission lines, so it is vital to masterminding each stage voltage with stage change, making them independent for each other.

Fig. 1 The flow chart of transmission line fault location.
Voltage traveling wave signal acquisition

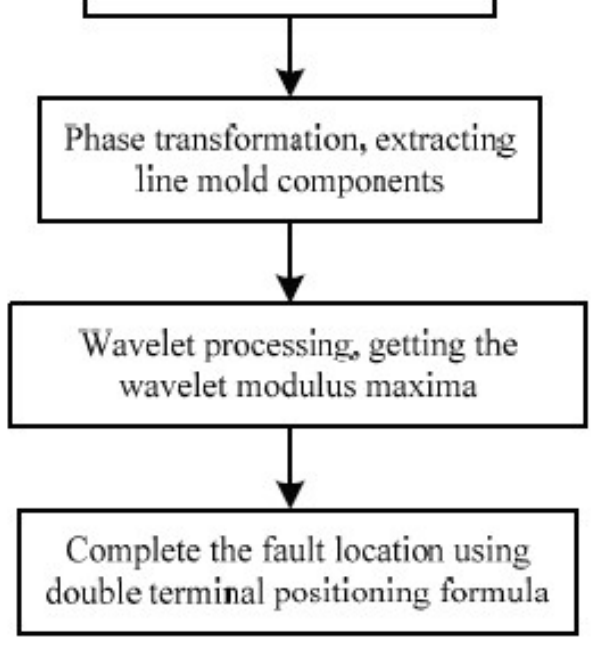

\section{B. Extracting Wave Front of Voltage Traveling Wave}

Double side positioning principle is based on initial traveling wave propagation time difference and wave velocity to determine fault location when the fault happening. 


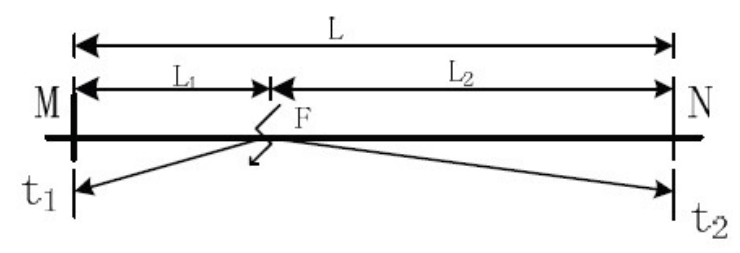

As appeared in figure 3, blame happens in F point, and the setting out wave spread to $\mathrm{M}$ and $\mathrm{N}$ focuses. The season of making a trip wave front coming to point $\mathrm{M}$ is $\mathrm{t} 1$, coming to point $\mathrm{N}$ is $\mathrm{t} 2$. Setting the entire length of line is $\mathrm{L}$, wave speed is $\mathrm{v}$, and the separation $\mathrm{F}$ to $\mathrm{M}, \mathrm{F}$ to $\mathrm{N}$ separately:

$$
\left\{\begin{array}{l}
L_{2}=\frac{L-\left(t_{1}-t_{2}\right) \cdot v}{2} \\
L_{1}=\frac{L-\left(t_{1}-t_{2}\right) \cdot v}{2}
\end{array}\right.
$$

\section{Single-ended fault location Algorithm}

- Single terminal methodologies are that the blame point is figured by the voyaging time between the principle section of the voyaging wave and the second arrival of the reflection wave at end of the line.

- This time is with respect to the blame detachment and the key is to look at the reflection system of voyaging wave. A relationship method is used to see the surge returning from the blame point and remember it from various surges present on the framework. The strategy is appropriate for a regular long queue, however unquestionably is lacking for a nearby in fault just a couple of kilometers from the estimating point.

\section{Double-ended fault location Algorithm}

- The twofold terminals strategies are that blame point is controlled by correctly time marking the section of voyaging wave at each complete of the line .This procedure depends less on building up assurance and framework running-way, et cetera... This methodology is used by and large.

- The speed is controlled by the scattered parameters ABCD of the line and commonly vacillates in the range $295-29 \mathrm{~m} / \mathrm{us}$ for $500 \mathrm{kV}$ line.

\section{CONCLUSION}

This paper proposed a calculation for distinguishing line flaws and grouping flaw composes in light of STFT in a transmission line. To check the execution of the proposed calculation, a $345 \mathrm{kV}$ transmission system was displayed utilizing EMTP-RV. Recreations were directed for ten sorts of line flaws. STFT was figured from the voltage motion after fault event. We checked that fault characterization utilizing our calculation is for the most part dependable. The reproduction comes about demonstrated that the proposed calculation can distinguish different faults in the transmission line. It can be utilized to react fittingly to different line issues. This paper likewise has examined the $\mathrm{T}$ transmission line fault area issue. In light of the voltage voyaging wave at the fault point, utilize phase change to separate line mode, utilize wavelet change to figure wavelet modulus maxima of the voltage voyaging wave and decide the entry time. As indicated by the functional $\mathrm{T}$ write transmission line, get the fault area equation.

\section{References}

1) Jun Han, Won-Ki Kim, Jae-Won Lee, Chul-Hwan Kim "FAULT TYPE CLASSIFICATION IN TRANSMISSION LINE USING STFT" [J].

2) Yongfu Li, Jin Fu, Qian Wang, Huadong Peng. Study on T type Transmission Line Fault Location Based on Voltage Traveling Wave Transmission Line Fault Location System [J]. State Grid Chongqing Electric Power Company Chongqing, China, 978-1-5090-0496-6/16 C2016 IEEE

3) Jianyin liu, Zhong Zheng "Fault Location and Type Identification on Transmission Line Using a Novel Traveling Wave Method" 2012 International Conference on High Voltage Engineering and Application, Shanghai, China, 978-1-4673-4746-4/12.

4) Han Kunlun, Cai Zexiang "Study on Protective Performance of HVDC Transmission Line Protection With Different Types of Line Fault" 978-1-4577-0365-2/11/\$26.00 (C2011 IEEE.

5) Don O. Koval, Ali Asraf Chowdhury "Assessment of Transmission-Line Common-Mode, StationOriginated, and Fault-Type Forced-Outage Rates" IEEE TRANSACTIONS ON INDUSTRY APPLICATIONS, VOL. 46, NO. 1, JANUARY/FEBRUARY 2010, 00939994/\$26.00 C 2010 IEEE. 
International Journal of Trend in Scientific Research and Development (IJTSRD) ISSN: 2456-6470

6) DENG Feng, ZENG Xiangjun "An Optimal Configuration Algorithm of Traveling Wave Fault Location Unit" 2011The International Conference on Advanced Power System Automation and Protection, 978-1-4244-9621-1/11/\$26.00 (C2011 IEEE.

7) J. D. Duan, B. H. Zhang "RESEARCH OF TRAVELING-WAVE DIFFERENTIAL BUS PROTECTION BASED ON WAVELET TRANSFORM" 2011.

8) Zhengyou $\mathrm{He}$, Ling $\mathrm{Fu}$, Sheng Lin "Fault Detection and Classification in EHV Transmission Line Based on Wavelet Singular Entropy" IEEE TRANSACTIONS ON POWER DELIVERY, VOL. 25, NO. 4, OCTOBER 2010, 0885$8977 / \$ 26.00$ C 2010 IEEE.

9) Ankit Jain, Beaulah Moses "SOFT COMPUTING BASED FAULT DETECTION \& CLASSIFICATION FOR TRANSMISSION LINES" International Conference on Electrical, Electronics, and Optimization Techniques (ICEEOT) - 2016, 978-1-4673-9939-5/16/\$31.00 (C)2016 IEEE.
10) Sago Tomaiii': and Simon Znidar "A Fast Recursive STFT Algorithm" 0-7803-31-095/96/\$5.00 1996 IEEE.

11) Yongfu Li, Jin Fu "Study on T type Transmission Line Fault Location Based on Voltage Traveling Wave" 978-1-5090-0496-6/16/\$31.00 (C2016 IEEE.

12) C. Subramani, A. A. Jimoh "Fault Investigation Methods on Power Transmission Line: A Comparative Study" 2016 IEEE PES Power Africa Conference, 978-1-4673-9981 4/16/\$31.00 (C)2016 IEEE.

13) L. Satish, Short Time Fourier and Wavelet Transformer Fault Detection in Power Transformer during the Impulse Tests IEE Proc.sci. Meas. Technol., Vol. 145, No. 2, March. 1998.

14) Papia Ray, Debani prasad Mishra "Hybrid Technique for Fault Location of a Distribution Line" IEEE INDICON 2015 1570160897, 978-14673-6540-6/15/\$31.00 C2015 IEEE.

15) HUI-BIN JIA, HAN-BO QIAN "A FAULT LOCATION METHOD IN DISTRIBUTION NETWORK WITH USE OF WAVELET-BASED TRAVELING-WAVE" 2012 International Conference on Wavelet Analysis and Pattern Recognition, Xian, 15-17 July, 2012, 978.1$4673 \cdot 1535 \cdot 7 / 121 \$ 31.00$ C2012 IEEE. 